\title{
Wave Packet Calculations of the Quantum Transport of Atoms through Nanoporous Membranes
}

\author{
Alfonso Gijón, José Campos-Martínez, and Marta I. Hernández* \\ Instituto de Física Fundamental, Consejo Superior de Investigaciones Científicas \\ (IFF-CSIC), Serrano 123, 28006 Madrid, Spain \\ E-mail: marta@iff.csic.es
}

\begin{abstract}
Quantum phenomena are relevant to the transport of light atoms and molecules through nanoporous membranes. Indeed, confinement provided by (sub-)nanometer pores enhances quantum effects such as tunneling and zero point energy (ZPE), even leading to quantum sieving of different isotopes of a given element. In this work we apply rigorous wave packet techniques to the simulation in the three-dimensional space of the passage of atoms through periodic rigid one-atom-thick membranes. We aim to assess more approximate quantum models such as one-dimensional tunneling calculations or transition state theory (TST). Calculations are reported for the transmission of ${ }^{3} \mathrm{He}$ and ${ }^{4} \mathrm{He}$ through graphdiyne as well as through a holey graphene model. For Hegraphdiyne, conclusions from tunneling-corrected TST are confirmed: both tunneling and ZPE effects are significant but competition between each other leads to a moderately small ${ }^{4} \mathrm{He} /{ }^{3} \mathrm{He}$ selectivity, in stark contrast with predictions from one-dimensional tunneling calculations. For the transport of He isotopes through leaky graphene, the computed transmission probabilities are highly structured suggesting widespread selective adsorption resonances and, this time, rate coefficients and selectivity ratios do
\end{abstract}


not agree well with estimations from TST. This study intends to go one step further towards a better understanding of the quantum-mechanical processes in the transport of gases through nanoporous membranes. 


\section{Introduction}

Recent progress in the fabrication of nanoporous two-dimensional membranes has led to propose them as efficient sieves at the molecular level. 1 - 3 Particularly, it has been suggested that these membranes could be used for the separation of a specific isotope within an atomic or molecular gas, $\frac{45}{45}$ a process which is both important and challenging. For example, ${ }^{3} \mathrm{He}$ is essential to several applications ranging from security to basic research but it is very rare and

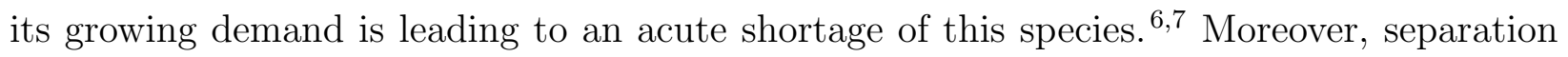
of ${ }^{3} \mathrm{He}$ from the much more abundant ${ }^{4} \mathrm{He}$-in turn, commonly extracted from natural gasusually involves very expensive cryogenic methods. Separation of the heavier isotopes of $\mathrm{H}_{2}$ is also crucial for various technologies. ${ }^{8}$ Ideally, single-layer membranes should involve a low energy consumption and efficiency, provided that the pores are designed to optimize the desired separation process.

For sufficiently low pressures, the study of purification of a gas mixture can be modeled by the dynamics of an atom or molecule passing through a pore of the membrane. In this way, rate coefficients for the transmission of the isotopic species of the mixture, say $k_{a}$ and $k_{b}$, are independently computed at a given temperature $T$, and the efficiency for the isotope separation is estimated by means of the selectivity ratio $S_{a / b}(T)=k_{a}(T) / k_{b}(T)$. Since isotopes are chemically identical, the separation mechanism must be provided by massdependent dynamical effects. At sufficiently low temperatures, quantum effects may entail large selectivity ratios as compared with those based on classical diffusion, for instance. Recently, a rather large number of works have shown that quantum tunneling might rule an efficient separation process. $\frac{59 \sqrt{14}}{4}$ However, these studies are based on one-dimensional (1D) quantum-mechanical calculations, whereas the molecular motion actually occurs in the three-dimensional (3D) space where differences between the quantum energy levels of the isotopes confined within the pores can also lead to quantum sieving. ${ }^{15}$ These features, which may be generally termed as zero point energy (ZPE) effects, $\frac{16}{16}$ have been invoked in various theoretical ${ }^{2 / 17+19}$ and experimental ${ }^{20 \mid 21}$ works on porous materials. It is worth to 
emphasize that tunneling and ZPE effects work in opposite directions: while tunneling favors the passage of lighter molecules, ZPE facilitates the transmission of the heavier ones since their smaller ZPE involves a smaller "effective size". Therefore, it is important to evaluate as accurately as possible the relative role of each effect on the systems of interest.

We have recently studied ${ }^{[22}$ the interplay between tunneling and ZPE effects in the transmission of He isotopes through graphdiyne, $\stackrel{23 \mid 24}{a}$ a promising new material for molecular separation applications $\frac{1125}{27}$ as it exhibits regularly distributed pores of sub-nanometer size. To this end we relied on transition state theory (TST) which takes into account the ZPE of the transition state (atom at the pore center) and to which reaction-path tunneling corrections were added. He-graphdiyne interactions were represented by a force field validated

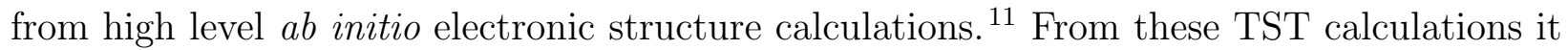
was found that ZPE dominates over tunneling in the studied temperature range (20-100 K): the ${ }^{4} \mathrm{He}$ transmission rate is therefore larger than the ${ }^{3} \mathrm{He}$ one, the selectivity ratio $k^{4} \mathrm{He} / k_{3} \mathrm{He}$ reaching $\approx 2.5$ at $20 \mathrm{~K}$. This is in sharp contrast with calculations considering just tunneling within a one-dimensional model: $\frac{1122}{122}$ since this model neglects the ZPE along the degrees of freedom perpendicular to the reaction path, ${ }^{3} \mathrm{He}$ transmission is always dominant and at $20 \mathrm{~K}$ the selectivity ratio is also $\approx 2.5$ but incorrectly favoring ${ }^{3} \mathrm{He}$ instead of ${ }^{4} \mathrm{He}$. Similar results have been reached using $1 \mathrm{D}$ models on related systems. ${ }^{9}$ It becomes clear that the transmission of atoms through nanopores must be studied with treatments incorporating all possible quantum effects and that more accurate quantum calculations may be needed to check the results from TST.

In this work we report rigorous quantum-mechanical calculations of the transmission of an atom through a periodic rigid one-atom-thick membrane. The present application combines wave packet techniques for the scattering of atoms by periodic surfaces ${ }^{28}$ with reaction flux methods 29130 to obtain transmission probabilities and rate coefficients. These threedimensional wave packet (WP3D in short) calculations are performed for He-graphdiyne ${ }^{11}$ as well as for a holey graphene model. $\frac{31}{10}$ In this way, commonly used simpler models can be 
tested by comparison with the present treatment. We also expect that this approach serves to uncover new clues for the proposal of optimal pores for quantum sieving.

The rest of the paper arranges as follows. In the following Section we give the theory for the transmission of a 3D wave packet through a periodic membrane, accompanied by a refresher outline of TST. Results are presented and discussed in Section III, first for Hegraphdiyne, followed by He-holey graphene. The report ends with a conclusion paragraph in Section IV.

\section{Transmission of an atom through a periodic mem-}

\section{brane: theoretical approach}

\section{Three-dimensional wave packet (WP3D) propagation}

In the present WP3D approach we consider the scattering of an atom of mass $\mu$ by a periodic non-vibrating membrane by means of time-dependent wave packet methods. ${ }^{32133}$ The membrane coincides with the $x y$ plane of the reference frame, whose origin is set at the center of one of its pores; hence the position of the atom is given by $\mathbf{r}=(\mathbf{R}, z), z$ being the distance to the membrane plane and $\mathbf{R}=(x, y)$. The wave packet representing the atom is discretized on a grid of evenly spaced $\mathbf{r}$ points and at the start of the propagation is given as a product of a Gaussian wave packet ${ }^{34}$ in $z$ times a plane wave with wave vector $\mathbf{K}$ in $\mathbf{R}$. Thus, as in the original work by Yinnon and Kosloff, 28 the periodicity of the system is fully exploited by matching the size of the $(x, y)$ grid to that of the unit cell, $\left(\Delta_{x}, \Delta_{y}\right)$, while the values of the parallel wave vector $\mathbf{K}$ are restricted such that the initial plane wave is commensurate with the membrane lattice. The wave packet is propagated in time subject to the timedependent Schrödinger equation, using the split operator method,,$\sqrt[32]{ }$ and is damped ${ }^{35136}$ in the asymptotic regions to avoid artificial reflections from the grid borders. To obtain the probability of transmission of the atom through the membrane, its is convenient to write first 
the asymptotic behavior of the stationary wave function for a translational energy $E=\frac{\hbar^{2} k^{2}}{2 \mu}$,

$$
\begin{aligned}
\Psi_{E}^{+}(\mathbf{r}) & \longrightarrow \sqrt{\frac{\mu}{2 \pi \Delta_{x} \Delta_{y} \hbar^{2}}}\left[\frac{e^{i \mathbf{k} \cdot \mathbf{r}}}{\sqrt{-k_{z}}}+\sum_{\mathbf{G}} A_{\mathbf{G}}^{+} \frac{e^{i\left[k_{z, \mathbf{G}}^{+} z+(\mathbf{K}+\mathbf{G}) \cdot \mathbf{R}\right]}}{\sqrt{k_{z, \mathbf{G}}^{+}}}\right] \\
& \longrightarrow \infty \\
z & \sqrt{\frac{\mu}{2 \pi \Delta_{x} \Delta_{y} \hbar^{2}}} \sum_{\mathbf{G}} A_{\mathbf{G}}^{-} \frac{e^{i\left[k_{z, \mathbf{G}}^{-} z+(\mathbf{K}+\mathbf{G}) \cdot \mathbf{R}\right]}}{\sqrt{-k_{z, \mathbf{G}}^{-}}}
\end{aligned}
$$

which represents an incident plane wave with a wave vector $\mathbf{k}=\left(k_{z}, \mathbf{K}\right)$ and a set of reflected $(+)$ and transmitted (-) waves with amplitudes $A_{\mathbf{G}}^{ \pm}$labeled by the reciprocal lattice vector, G. Note that the parallel wave vectors of these waves obey the Bragg condition whereas the perpendicular one is modified to satisfy the conservation of energy, $k_{z, \mathbf{G}}^{ \pm}= \pm\left[k^{2}-(\mathbf{K}+\mathbf{G})^{2}\right]^{1 / 2}$, as energy exchange with the membrane is neglected in the present approach. This function is normalized as $<\Psi_{E}^{+} \mid \Psi_{E^{\prime}}^{+}(\mathbf{r})>=\delta\left(E-E^{\prime}\right)$. It can be shown that the (total) transmission probability, which is the sum of the squared transmission amplitudes, can be also obtained from the flux of the stationary wave function through a surface $z=z_{f}$ separating transmitted from incident and reflected waves, 29

$$
\begin{aligned}
P_{\text {trans }}(E) & =\sum_{\mathbf{G}}\left|A_{\mathbf{G}}^{-}\right|^{2} \\
& =\frac{2 \pi \hbar^{2}}{\mu} \operatorname{Im}\left(\left.\int d x d y \Psi_{E}^{+*}\left(x, y, z_{f}\right) \frac{d \Psi_{E}^{+}}{d z}\right|_{z=z_{f}}\right) .
\end{aligned}
$$

We have employed this flux formula for computing $P_{\text {trans }}$, where $\Psi_{E}^{+*}\left(x, y, z_{f}\right)$ is obtained from the time-energy Fourier transform of the evolving wave packet. 30137

The transmission rate coefficient is then obtained from the integration of $P_{\text {trans }}(E)$, properly weighted by the Boltzmann factor: 


$$
k(T)=\frac{1}{h Q_{\text {trans }}} \int e^{-E /\left(k_{B} T\right)} P_{\text {trans }}(E) d E,
$$

where $Q_{\text {trans }}=\left(2 \pi \mu k_{B} T / h^{2}\right)^{3 / 2}$ is the translational partition function per unit volume. In detail, $P_{\text {trans }}$ not only depends on the translational energy but it is also labeled by the parallel wave vector $\mathbf{K}$. A complete calculation of the rate coefficients should involve averaging over a sufficiently large set of $\mathbf{K}$ values. In this work we have used initial wave packets perpendicularly approaching the membrane $(\mathbf{K}=\mathbf{0})$ since this direction matches the minimun energy path. The effect of varying the angle of incidence $(\mathbf{K} \neq \mathbf{0})$ is briefly examined at the end of Section III.

Computational details of the WP3D simulations are provided in the Supporting Information Section.

\section{Transition state theory (TST)}

Rate coefficients computed in this way are compared with those obtained from TST as detailed in Ref. ${ }^{[22}$ In short, it is assumed that the reaction path is a straight line perpendicular to the membrane $(\mathbf{K}=\mathbf{0})$ and crossing the center of the pore, which is the TS. Hence the transmission rate coefficient can be written as ${ }^{\sqrt{1738139}}$

$$
k_{T S T}(T)=\gamma \frac{k_{B} T}{h} \frac{Q^{\ddagger}}{Q_{\text {trans }}} f_{\text {tunn }}(T),
$$

where

$$
Q^{\ddagger}=\sum_{n} e^{-E_{n} / k_{B} T}
$$

is the TS partition function, $E_{n}$ being the TS energy levels of the bound states for the degrees of freedom perpendicular to the reaction path (He in-plane vibrations inside the pore). In addition, $f_{\text {tunn }}(T)$ is a correction for tunneling effects along the reaction path.22138139 Finally, $\gamma$ is a correction factor (not considered previously) related to the fact that only a fraction of 
the membrane is effective for permeation $\underline{40}$ and is defined as

$$
\gamma=n_{p} \frac{A_{e f f}}{A_{u c}}
$$

where $A_{\text {eff }}$ is the effective size of the pore, $A_{u c}=\Delta_{x} \cdot \Delta_{y}$ is the area of the unit cell and $n_{p}$ is the number of pores per unit cell. In this way, $\gamma$ can be understood as a classical transmission probability for a membrane made of perfectly penetrable pores of area $A_{\text {eff }}$ surrounded by a completely impenetrable sheet. Present procedure for estimation of $A_{\text {eff }}$ is detailed below.

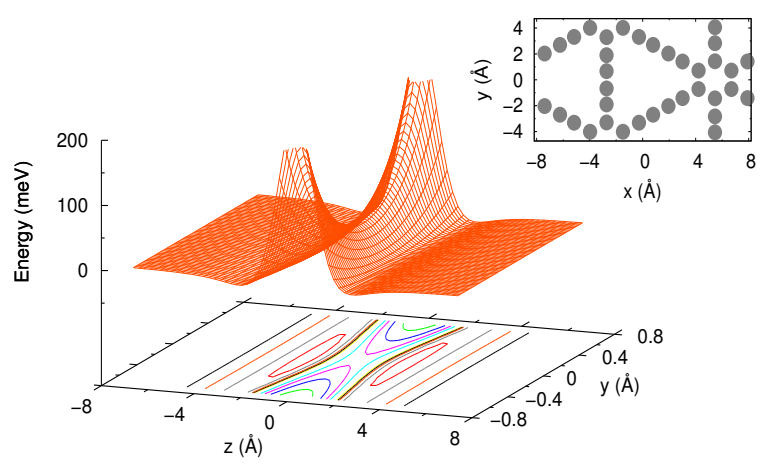

Figure 1: Right-upper panel: Graphdiyne unit cell employed in the WP3D calculations (carbon atoms are depicted by gray filled circles). Left-lower panel: He-graphdiyne interaction potential $(\mathrm{meV})$ as a function of the $y$ and $z$ coordinates, with $x=0$.

\section{Results and discussion}

\section{Transmission of He isotopes through graphdiyne.}

Our first choice for performing WP3D calculations is the transmission of He isotopes through graphdiyne, as for this system a reliable force field has been already obtained ${ }^{11}$ and TST selectivity ratios showing an involved behavior have been already reported.22 Briefly, the He-graphdiyne potential is obtained as a pairwise sum over He-C potentials represented

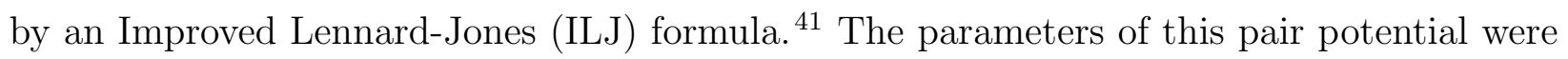


a)
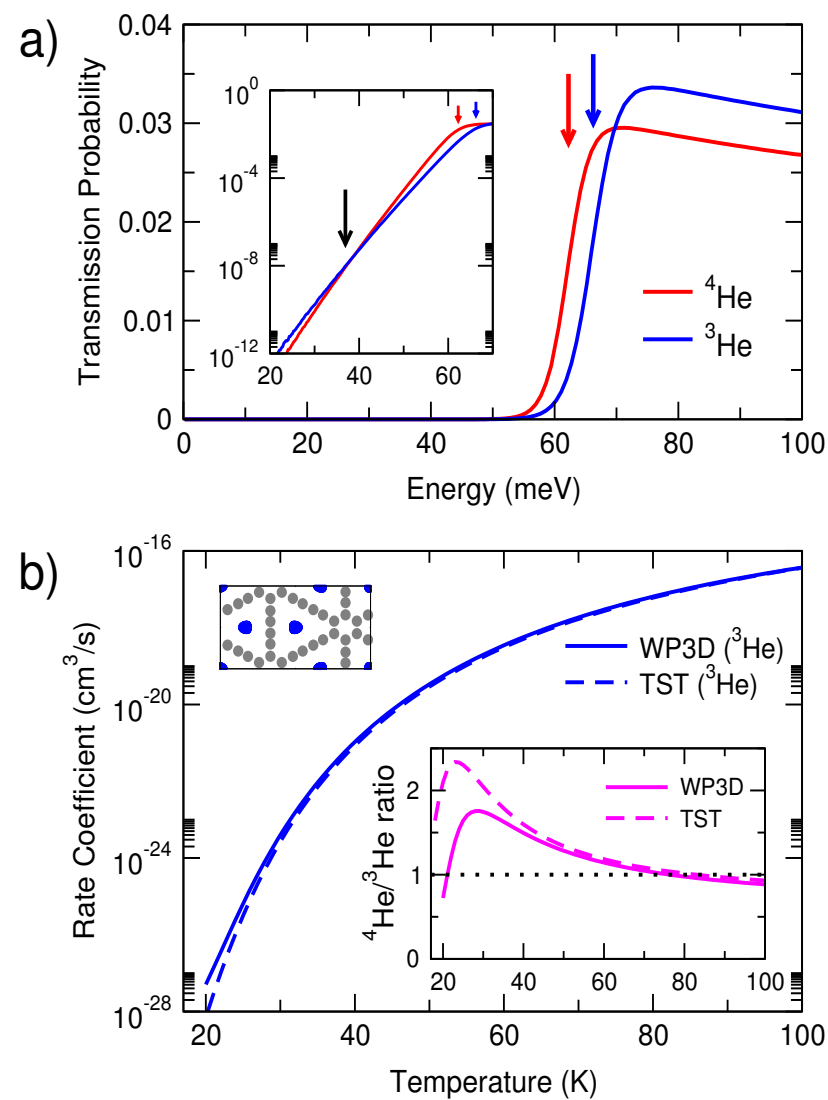

Figure 2: a) WP3D probabilities of ${ }^{4} \mathrm{He}$ and ${ }^{3} \mathrm{He}$ transmission through graphdiyne as a function of the translational energy of the atom (in meV). Red and blue arrows indicate the ${ }^{4} \mathrm{He}$ and ${ }^{3} \mathrm{He}$ reaction thresholds, respectively, as predicted by TST. ${ }^{22}$ The inset shows these probabilities (in logarithmic scale) for the low energy region. The black arrow shows the potential barrier height. b) ${ }^{3} \mathrm{He}$ WP3D rate coefficients vs. temperature compared with TST estimations. Graphdiyne unit cell, together with the effective area for ${ }^{3} \mathrm{He}$ transmission as obtained from TST (blue spots), are displayed in the upper-left inset. Finally, the ${ }^{4} \mathrm{He} /{ }^{3} \mathrm{He}$ selectivity vs. temperature is presented in the lower-right inset.

optimized from comparison with benchmark "coupled" supermolecular second-order MøllerPlesset perturbation theory ${ }^{42}$ calculations and using aug-cc-pVTZ and aug-cc-pV5Z basis sets for the carbon and helium atoms, respectively, as described in detail elsewhere.11] Graphdiyne unit cell [with lengths $\left(\Delta_{x}, \Delta_{y}\right)=(16.37 \AA, 9.45 \AA)$ ] is depicted in the rightupper panel of Fig,1. A detailed description of the geometry of the membrane is given in Ref. ${ }^{111}$ In the left-lower panel of the same figure, a plot of the He-graphdiyne potential is presented. The point $\mathbf{r}=0$ is a saddle: whereas it corresponds to the maximum of a barrier potential along the "reaction-path" $z$ coordinate -with a height of $E_{0}=36.92 \mathrm{meV}$ - it is a 
minimum with respect to displacements along the $y$ and $x$ "in-pore" degrees of freedom. It is worthwhile to note that while the potential barrier is rather low at this saddle point it rapidly rises for paths different to the minimum energy path.

Transmission probabilities for ${ }^{4} \mathrm{He}$ and ${ }^{3} \mathrm{He}$ are presented and compared in Fig, 2.a). These probabilities rise above $60 \mathrm{meV}$, a value much higher than the potential barrier. The positions of these thresholds agree quite well with the TST prediction (indicated by arrows in the figure): they correspond to the sum of the potential barrier $(36.92 \mathrm{meV})$ and the ZPE at TS (25.32 and $29.31 \mathrm{meV}$ for ${ }^{4} \mathrm{He}$ and ${ }^{3} \mathrm{He}$, respectively $\left.{ }^{222}\right)$, leading to $62.24\left({ }^{4} \mathrm{He}\right)$ and 66.23 $\left({ }^{3} \mathrm{He}\right) \mathrm{meV}$. In addition, it is worth noticing that, after threshold, probabilities just raise to slightly below (above) 0.03 for ${ }^{4} \mathrm{He}\left({ }^{3} \mathrm{He}\right)$. Assuming that this value is equal to the classical probability given by $\gamma$ (Eq. 6) and using that equation with $n_{p}=4$, the effective pore size can be estimated to be $A_{\text {eff }} \approx 1.2 \AA^{2}$. Finally, the behavior below threshold is shown in the inset of Fig. 22a), where it can be seen that the probabilities decrease exponentially as energy decreases. There the black arrow indicates the potential barrier. Nearly below this energy ${ }^{3} \mathrm{He}$ transmission becomes more probable, in agreement with previous 1D calculations ${ }^{11122}$ where, below the barrier, the lighter atom exhibits a larger tunneling probability.

Rate coefficients as functions of temperature are determined from these probabilities (Eq.3) and the result for ${ }^{3} \mathrm{He}$ is presented in Fig,2.b) for the temperature range 20-100 K. Rate coefficient calculations were not attempted at lower temperatures because probabilities at low translational energies $(<20 \mathrm{meV})$ are so small that they become difficult to converge. The error in the rate coefficients associated to these uncertainties is estimated to be below $3 \%$ at $T=20 \mathrm{~K}$ and rapidly decreases as temperature increases. These WP3D rate coefficients are compared with those previously reported within TST $^{222}$ except that in this work we additionally include the correction given by $\gamma$ (Eqs. 4 and 6). To that end, we have chosen to estimate $A_{\text {eff }}$ from the ground state TS wave function, $\Psi_{0}(x, y)$, as the region where $\left|\Psi_{0}(x, y)\right|^{2}$ is larger than a given cutoff, $f_{\text {cut }}$. This cutoff have been set ad hoc to fit the $\gamma$ factor derived above from the WP3D calculations, i.e., taking $f_{\text {cut }}=10^{-4}$ leads to 
$\gamma(T S T)=0.033$ and 0.029 for ${ }^{3} \mathrm{He}$ and ${ }^{4} \mathrm{He}$, respectively, a result that matches the WP3D probabilities for the two isotopic species. In particular, notice that $\gamma(T S T)$ is larger for ${ }^{3} \mathrm{He}$, in agreement with the behavior of the probabilities. In this way, despite the procedure is not straightforward, it does suggest a nice relationship between the spatial extension of the TS wave function and the effective size of the pore. With this $\gamma(T S T)$, WP3D and TST rate coefficients agree very well along the whole temperature range, except at the lowest temperatures where the WP3D coefficients are somewhat larger, probably due to an underestimation of tunneling from TST. For ${ }^{4} \mathrm{He}$ (not shown) WP3D and TST comparison is even more successful. Finally, the ${ }^{4} \mathrm{He} /{ }^{3} \mathrm{He}$ selectivity (ratio of rate coefficients) is reported in the inset of Fig 2.b). It can be seen that there is a fairly good agreement between the WP3D and TST calculations, although below $40 \mathrm{~K}$ WP3D calculations show that the preference for the transport of the heavier isotope is not as significant as originally predicted by TST.

WP3D calculations confirm the conclusions previously drawn from the TST calculations: $\stackrel{22]}{2}$ both tunneling and zero point energy are very important effects in the permeability of He at low temperatures but, as they operate in opposite directions, in the end we cannot achieve a large difference between the transmission rates of the two isotopes. We would like to stress that neglect of one or the other quantum effect in the model would have led to qualitatively erroneous results. Moreover, the very low values of the related rate coefficients

(Fig. 2.b)) suggest that the actual flux of these species through the sieve would be extremely

slow. It is well known that permeability usually decreases as selectivity increases. ${ }^{[3]}$ As a possible strategy for achieving isotope separation, new membranes could be designed where one of the two competing quantum effects are suppressed while the flux of the more permeable species is kept sufficiently large. With that aim, we report below results for a model system where tunneling is in principle absent.

\section{Transmission of He isotopes through a holey graphene model (P7)}

We have adopted the model of Sun et al, ${ }^{31}$ where nanopores are generated by eliminating 
a)

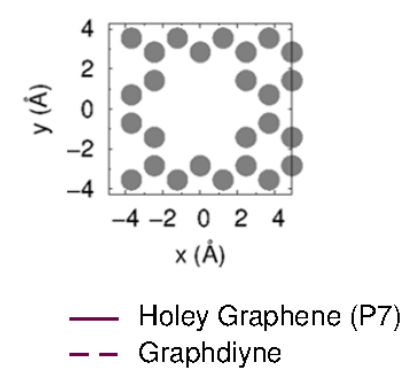

b)
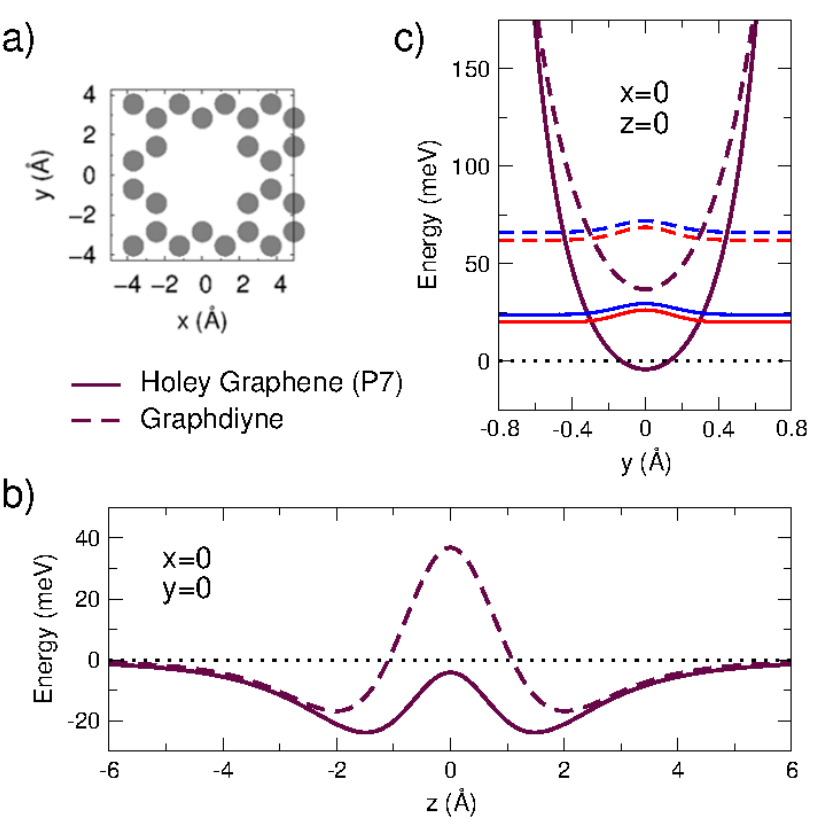

.
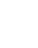
this difference as well as the suppression of tunneling which would favor ${ }^{3} \mathrm{He}$. Interestingly, as these effective barriers are much lower than those of He-graphdiyne, we foresee that the transmission rates will be much larger in the present system.

\section{a)}

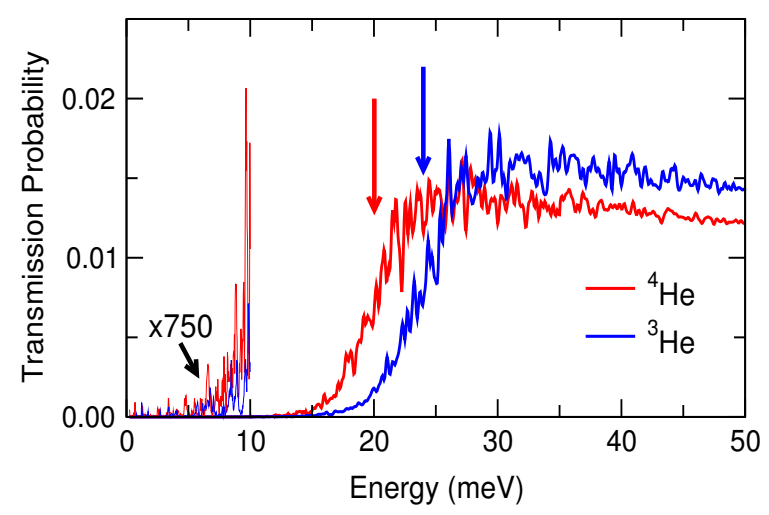

b)

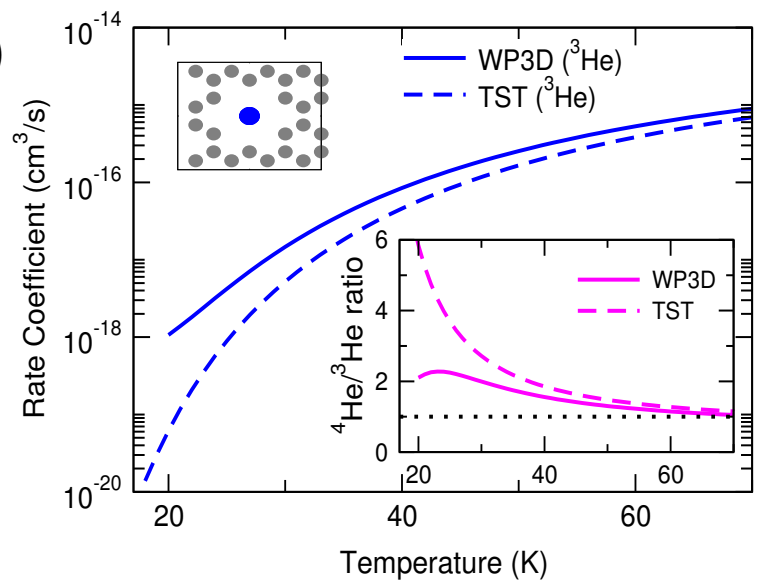

Figure 4: a) WP3D transmission probabilities of ${ }^{4} \mathrm{He}$ and ${ }^{3} \mathrm{He}$ through the $\mathrm{P} 7 \mathrm{membrane}$ vs. kinetic energy of the atom. Red (blue) arrow indicates the ${ }^{4} \mathrm{He}\left({ }^{3} \mathrm{He}\right)$ transmission threshold as predicted by TST. For energies below $10 \mathrm{meV}$ probabilities are shown magnified by a factor of 750 . b) ${ }^{3} \mathrm{He}$ WP3D rate coefficients vs. temperature compared with TST estimations. P7 unit cell, including the TST effective area for ${ }^{3} \mathrm{He}$ transmission, are displayed in the upperleft inset. Finally, the ${ }^{4} \mathrm{He} /{ }^{3} \mathrm{He}$ selectivity vs. temperature is presented in the lower-right inset.

He-P7 transmission probabilities are presented in Fig 4 a). As expected, the probabilities rise at translational energies close to the TST estimation given by the sum of the ZPE and the potential energy, leading to $20.03\left({ }^{4} \mathrm{He}\right)$ and $23.99 \mathrm{meV}\left({ }^{3} \mathrm{He}\right)$ (values indicated by arrows in the figure). However and in contrast to He-graphdiyne, probabilities exhibit a multitude of peaks along the whole energy range. We have checked that these structures 
are not due to any artifact in the calculations. We believe that these peaks correspond to selective adsorption resonances, a process that can be understood as a temporal trapping of the incident wave into a bound state of the laterally averaged potential while the motion along the parallel coordinates, ruled by the Bragg condition, becomes faster for the sake of the conservation of energy. $\frac{446}{46}$ Analysis of the wave packet propagation supports this argument: it is noticed that after the main portion of the wave packet has been either reflected or transmitted by direct scattering, a non-negligible fraction of this wave packet remains trapped along the adsorption region $(\approx 3 \AA)$ for a long time while it is slowly decaying towards the reflection and the transmission regions. Resonance structures are not seen in the He-graphdiyne transmission probabilities probably because they are extremely narrow and/or the probability of formation of resonances during the wave packet simulation is very small. We plan to get more insight into the role played by these resonances from simulations of the decaying of initially prepared adsorbed states. $\underline{46}$

WP3D rate coefficients for ${ }^{3} \mathrm{He}-\mathrm{P} 7$ as functions of temperature are reported in Fig.4.b). Comparing with graphdiyne (Fig, 2 b)), it is worth noticing their large absolute values which are due to the lower thresholds in the transmission probabilities. TST calculations have been also performed for this system to test whether this theory can predict the WP3D results. TST rate coefficients were obtained using Eq. 4 where, in this case, $f_{\text {tunn }}=1$, as the minimum energy path is barrier-less. The effective pore size, $A_{\text {eff }}$, has been computed, as for the previous system, from the TS ground state wave function and taking the same value for $f_{\text {cut }}$, leading to $A_{\text {eff }}=1.1\left({ }^{4} \mathrm{He}\right)$ and $1.2\left({ }^{3} \mathrm{He}\right) \AA^{2}$. Taking into account that $n_{p}=$ 1 for this unit cell, the resulting $\gamma(T S T)$ values are 0.0130 and 0.0144 for ${ }^{4} \mathrm{He}$ and ${ }^{3} \mathrm{He}$, respectively, which are in very good agreement with the heights of the plateaus reached for the probabilities in the higher energy region (Fig,4a)). However, TST rate coefficients do not agree so well with the WP3D calculations, especially at low temperatures where differences become of almost two orders of magnitude. TST also underestimates the rate coefficients of ${ }^{4} \mathrm{He}-\mathrm{P} 7$ (not shown) although to a lesser extent. As a consequence, TST is unable to 
predict the qualitative behavior of the ${ }^{4} \mathrm{He} /{ }^{3} \mathrm{He}$ selectivity ratio, reported in the inset of Fig.4. b). In fact, while TST predicts an increase of the ${ }^{4} \mathrm{He} /{ }^{3} \mathrm{He}$ selectivity as temperature decreases (reaching a promising value of six at $20 \mathrm{~K}$ ), the maximum selectivity reached from the accurate treatment is just 2.3 (at about $23 \mathrm{~K}$ ). Tunneling (either direct or mediated by the resonances ${ }^{47}$ ) can be at the origin of the larger values of the WP3D rate coefficients. The fact that discrepancies are larger for the lighter isotope supports this argument. Therefore, it appears that tunneling still operates for this system and competes with ZPE effects so that, after all, the quantum sieving is not as important as initially expected based on simpler models.

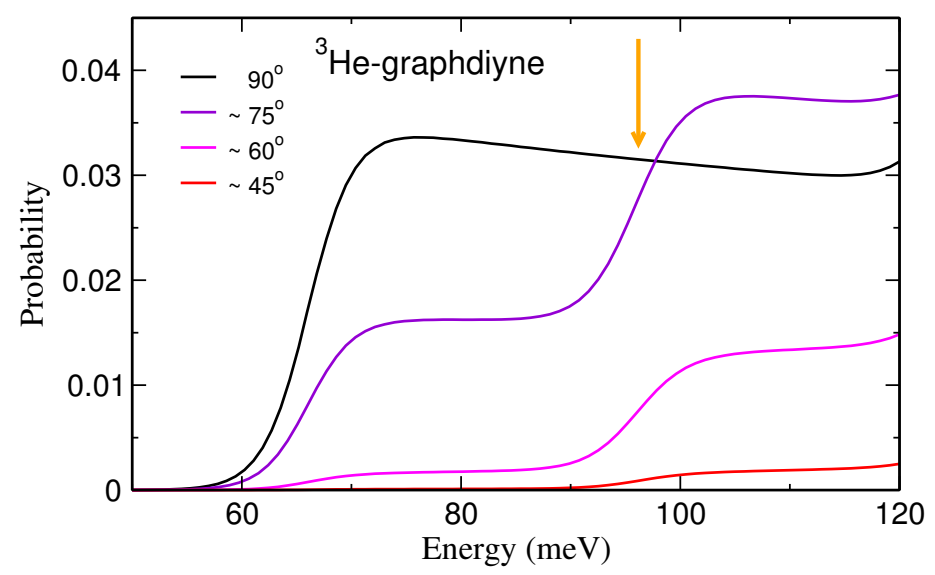

Figure 5: WP3D transmission probabilities for ${ }^{3} \mathrm{He}$-graphdiyne as functions of kinetic energy $(\mathrm{meV})$ for initial wave packets with a distribution of incidence angles $\theta=\arctan \left(k_{z} / K_{y}\right)$ with central values $\theta=75$ (violet), 60 (magenta) and 45 (red) degrees, compared with the transmission probability for normal incidence. Orange arrow indicates the energy of the first excited TS. See text for details.

Before finishing, we would like to briefly explore on the effect of choosing incidence directions of the wave packets different from the perpendicular one. To this end, the $y$ component of the initial parallel wave vector has been set to values different from zero while matching the periodicity of the lattice, i.e., $K_{y}=2 \pi l / \Delta_{y}$, where $l$ is an integer number. Several $l$ values where chosen so that $\arctan \left(k_{z 0} / K_{y}\right)$ equals the desired incidence angle, $\theta$, where $k_{z 0}$ is the central wave vector of the initial Gaussian ${ }^{34}$ wave packet along the $z$ direction, $G\left(z ; z_{0}, k_{z 0}, \alpha\right)$ (see Eqs.1 and 2 of Supporting Information). It must be noted 
that this angle just indicates an average incidence direction, as the mentioned wave packet involves a distribution of $k_{z}$ 's and therefore, a distribution of incidence angles (except for normal incidence). Probabilities for transmission of ${ }^{3} \mathrm{He}$ through graphdiyne are presented in Fig. 5 for central angles $\theta=75,60$ and 45 degrees, corresponding to $l=4,8$ and 11, respectively, and compared with normal incidence (setting $z_{0}=12 \AA, k_{z 0}=10.82 \sin (\theta) \AA^{-1}$, and $\alpha=0.388$ a.u.). It is noted first that the probability for transmission decreases quite fast as soon as the incidence angle departures from the perpendicular direction. Second, all probabilities rise around the same translational energy $(\approx 66 \mathrm{meV})$, but for the wave packets not impinging the surface perpendicularly, a second "step" in the probability shows at higher energies. This feature can be understood as the population of the first excited transition state. Indeed, the energy of this state is $59.26 \mathrm{meV}^{22}$ which, added to the potential barrier gives $96.18 \mathrm{meV}$ (orange arrow), in coincidence with the second transmission threshold. In detail, there are two degenerated states at this energy, one antisymmetric with respect to the $x$ axis and the other, with respect to the $y$ axis. ${ }^{22}$ This feature might be at the origin of the absence of a step in the probability for normal incidence: an initial wave packet with $l=0$ is constant with respect to $x$ and $y$ and hence it is unable to populate an antisymmetric transition state. On the contrary, if the initial state involves a periodic function it can eventually match the symmetry of the TS as it evolves in time. Finally, another step in the transmission probabilities is guessed at about $120 \mathrm{meV}$ and indeed TST predicts ${ }^{22} \mathrm{a}$ subsequent threshold at 127-128 meV (with three nearly degenerated states of different symmetries).

\section{Concluding remarks}

We have reported a three-dimensional wave packet approach for the study of the passage of atoms through nanoporous one-atom-thick membranes, in this way improving on previous theoretical studies. Computed transmission probabilities of He isotopes through graphdiyne 
and a leaky graphene model indicate the relevance of quantum effects such as tunneling, transition state zero point energy and, as a novelty, selective adsorption resonances. However, the two membranes studied are not efficient enough for practical isotope separation purposes, so more should be learned on the interactions and transport dynamics for the design of useful quantum sieving systems.

Transmission probabilities and rate coefficients are very sensitive to the He-C force field employed since a very small variation of the pair potential parameters can largely affect the features of the interaction potential near the pore center. Test calculations using transition state theory on the He-holey graphene model show that a decrease of a few percent in the $\sigma$ Lennard-Jones parameter can reduce the energy thresholds for transmission by $50 \%$, with a subsequent substantial increase of the rate coefficients. The effect on the selectivity ratios is expected to be milder, as also discussed in a related study. ${ }^{22}$ Nevertheless, wave packet calculations should be carried out to confirm these trends. Concerning He-graphdiyne, we are confident that (except for effects due to membrane vibrations) present results are reliable since the Improved Lennard Jones force field employed reproduces very accurately high level ab initio calculations, specifically, for the critical region around the center of the pore. $\frac{11222}{112}$

Regarding the comparison with simpler models, it is verified that one-dimensional quantum calculations lead to incorrect selectivity ratios because they neglect concomitant zeropoint energy effects for the degrees of freedom perpendicular to the reaction path. In addition, transition state theory is found to be successful for He-graphdiyne but fails for the holey graphene model. More studies are needed to assess the conditions for the validity of this theory. Moreover, it is feasible to extend the present approach to more complex systems such as diatoms or multilayered membranes, for instance. However, description of membrane vibrations and their coupling to the atomic motion remains an important but demanding task, although much progress is being achieved in the theoretical treatment of related problems. $\frac{48}{4}$ 


\section{Acknowledgments}

We thank Dr. Massimiliano Bartolomei for helpful discussions and a critical reading of the manuscript. The work has been funded by Spanish MINECO grant FIS2013-48275-C2-1-P. Allocation of computing time by CESGA (Spain) and support by the COST-CMTS Action CM1405 "Molecules in Motion (MOLIM)" are also acknowledged.

\section{Supporting Information}

Computational details of the three-dimensional wave packet calculations for the transmission of atoms through periodic membranes.

\section{References}

(1) Koenig, S. P.; Wang, L.; Pellegrino, J.; Bunch, J. S. Selective Molecular Sieving through Porous Graphene. Nature Nanotechnology 2012, 7, 728-732.

(2) Jiao, Y.; Du, A.; Hankel, M.; Smith, S. C. Modelling Carbon Membranes for Gas and Isotope Separation. Phys. Chem. Chem. Phys. 2013, 15, 4832-4843.

(3) Huang, L.; Zhang, M.; Li, C.; Shi, G. Graphene-Based Membranes for Molecular Separation. J. Phys. Chem. Lett. 2015, 6, 2806-2815.

(4) Schrier, J. Helium Separation Using Porous Graphene Membranes. J. Phys. Chem. Lett. 2010, 1, 2284-2287.

(5) Hauser, A. W.; Schwerdtfeger, P. Nanoporous Graphene Membranes for Efficient ${ }^{3} \mathrm{He} /{ }^{4} \mathrm{He}$ Separation. J. Phys. Chem. Lett. 2012, 3, 209-213.

(6) Cho, A. Helium-3 Shortage Could Put Freeze on Low-Temperature Research. Science 2009, 326, 778-779. 
(7) Nuttall, W. J.; Clarke, R. H.; Glowacki, B. A. Resources: Stop Squandering Helium. Nature 2012, 485, 573-575.

(8) Cai, J.; Xing, Y.; Zhao, X. Quantum Sieving: Feasibility and Challenges for the Separation of Hydrogen Isotopes in Nanoporous Materiasls. $R S C$ Advances 2012, 2, 85798586.

(9) Hauser, A. W.; Schrier, J.; Schwerdtfeger, P. Helium Tunneling through NitrogenFunctionalized Graphene Pores: Pressure- and Temperature-Driven Approaches to Isotope Separation. J. Phys. Chem. C 2012, 116, 10819-10827.

(10) Mandrà, S.; Schrier, J.; Ceotto, M. Helium Isotope Enrichment by Resonant Tunneling through Nanoporous Graphene Bilayers. J. Phys. Chem. A 2014, 118, 6457-6465.

(11) Bartolomei, M.; Carmona-Novillo, E.; Hernández, M. I.; Campos-Martínez, J.; Pirani, F.; Giorgi, G. Graphdiyne Pores: "Ad Hoc" Openings for Helium Separation Applications. J. Phys. Chem. C 2014, 118, 29966-29972.

(12) Lalitha, M.; Lakshmipathi, S.; Bhatia, S. K. Defect-Mediated Reduction in Barrier for Helium Tunneling through Functionalized Graphene Nanopores. J. Phys. Chem. C 2015, 119, 20940-20948.

(13) Li, F.; Qu, Y.; Zhao, M. Efficient Helium Separation of Graphitic Carbon Nitride Membrane. Carbon 2015, 95, 51-57.

(14) Qu, Y.; Li, F.; Zhou, H.; Zhao, M. Highly Efficient Quantum Sieving in Porous Graphene-like Carbon Nitride for Light Isotopes Separation. Scientific Reports 2016, 6,19952 .

(15) Beenakker, J. J. M.; Borman, V. D.; Krylov, S. Y. Molecular Transport in Subnanometer Pores: Zero-Point Energy, Reduced Dimensionality and Quantum Sieving. Chem. Phys. Lett. 1995, 232, 379-382. 
(16) We employ the term "ZPE effects" to refer in general to the effects due to the quantization of the states within the pore (identified as the transition state), and more especifically, to the in-pore ground state as it is the most populated state at low temperatures.

(17) Hankel, M.; Zhang, H.; Nguyen, T. X.; Bhatia, S. K.; Gray, S. K.; Smith, S. C. Kinetic Modelling of Molecular Hydrogen Transport in Microporous Carbon Materials. Phys. Chem. Chem. Phys. 2011, 13, 7834-7844.

(18) Kumar, A. V. A.; Bathia, S. K. Quantum Effect Induced Reverse Kinetic Molecular Sieving in Microporous Materials. Phys. Rev. Lett. 2005, 95, 245901.

(19) Schrier, J.; McClain, J. Thermally-Driven Isotope Separation across Nanoporous Graphene. Chem. Phys. Lett. 2012, 521, 118-124.

(20) Zhao, X.; Villar-Rodil, S.; Fletcher, A. J.; Thomas, K. M. Kinetic Isotope Effect for $\mathrm{H}_{2}$ and $\mathrm{D}_{2}$ Quantum Molecular Sieving in Adsorption/Desorption on Porous Carbon Materials. J. Phys. Chem. B 2006, 110, 9947-9955.

(21) Nguyen, T. X.; Jobic, H.; Bathia, S. K. Microscopic Observation of Kinetic Molecular Sieving of Hydrogen Isotopes in a Nanoporous Material. Phys. Rev. Lett. 2010, 105, 085901.

(22) Hernández, M. I.; Bartolomei, M.; Campos-Mertínez, J. Transmission of Helium Isotopes through Graphdiyne Pores: Tunneling versus Zero Point Energy Effects. J. Phys. Chem. A 2015, 119, 10743-10749, (Note that, the rate coefficients computed and shown in Fig. 4 therein were erroneously multiplied by $(2 \pi)^{-3 / 2}$. This error does not affect either the rest of the results nor the conclusions of the work.).

(23) Li, G.; Li, Y.; Liu, H.; Guo, Y.; Li, Y.; Zhu, D. Architecture of Graphdiyne Nanoscale Films. Chem. Commun. 2010, 46, 3256-3258. 
(24) Zhou, J.; Gao, X.; Liu, R.; Xie, Z.; Yang, J.; Zhang, S.; Zhang, G.; Liu, H.; Li, Y.; Zhang, J.; Liu, Z. Synthesis of Graphdiyne Nanowalls Using Acetylenic Coupling Reaction. J. Am. Chem. Soc. 2015, 137, 7596-7599.

(25) Jiao, Y.; Du, A.; Hankel, M.; Zhu, Z.; Rudolph, V.; Smith, S. C. Graphdiyne: A Versatile Nanomaterial for Electronics and Hydrogen Purification. Chem. Commun. 2011, 47, 1184311845.

(26) Cranford, S. W.; Buehler, M. J. Selective Hydrogen Purification through Graphdiyne under Ambient Temperature and Pressure. Nanoscale 2012, 4, 4587-4593.

(27) Bartolomei, M.; Carmona-Novillo, E.; Hernández, M. I.; Campos-Martínez, J.; Pirani, F.; Giorgi, G.; Yamashita, K. Penetration Barrier of Water through Graphynes' Pores: FirstPrinciples Predictions and Force Field Optimization. J. Phys. Chem. Lett. 2014, 5, 751-755.

(28) Yinnon, A. T.; Kosloff, R. A Quantum-Mechanical Time-Dependent Simulation of the Scattering from a Stepped Surface. Chem. Phys. Lett. 1983, 102, 216-223.

(29) Miller, W. H. Quantum Mechanical Transition State Theory and a New Semiclassical Model for Reaction Rate Constants. J. Chem. Phys. 1974, 61, 1823-1834.

(30) Zhang, D.; Zhang, J. Z. H. Full-Dimensional Time-Dependent Treatment for Diatom-Diatom Reactions: The $\mathrm{H}_{2}+\mathrm{OH}$ Reaction. J. Chem. Phys. 1991, 101, 1146-1156.

(31) Sun, C.; Boutilier, M. S. H.; Au, H.; Poesio, P.; Bai, B.; Karnik, R.; Hadjiconstantinou, N. G. Mechanisms of Molecular Permeation through Nanoporous Graphene Membranes. Langmuir 2014, 30, 675-682.

(32) Feit, M. D.; Fleck, J. A.; Steiger, A. Solution of the Schrödinger Equation by a Spectral Method. J. Comput. Phys. 1982, 47, 412-433.

(33) Kosloff, D.; Kosloff, R. A Fourier Method Solution for the Time Dependent Schrödinger Equation as a Tool in Molecular Dynamics. J. Comp. Phys. 1983, 52, 35-53. 
(34) Heller, E. J. Time-Dependent Approach to Semiclassical Dynamics. J. Chem. Phys. 1975, 62, 1544-1555.

(35) Heather, R.; Metiu, H. An Efficient Procedure for Calculating the Evolution of the Wave Function by Fast Fourier Transform Methods for Systems with Spatially Extended Wave Function and Localized Potential. J. Chem. Phys. 1987, 86, 5009-5017.

(36) Pernot, P.; Lester, W. A. Multidimensional Wave-Packet Analysis: Splitting Method for Time-Resolved Property Determination. Int. J. Quantum Chem. 1991, 40, 577-588.

(37) di Domenico, D.; Hernández, M. I.; Campos-Martínez, J. A Time-Dependent Wave Packet Approach for Reaction and Dissociation in $\mathrm{H}_{2}+\mathrm{H}_{2}$. Chem. Phys. Lett. 2001, 342, 177-184.

(38) Truhlar, D. G.; Kuppermann, A. Exact and Approximate Quantum Mechanical Reaction Probabilities and Rate Constants for the Collinear $\mathrm{H}+\mathrm{H}_{2}$ Reaction. J. Chem. Phys. 1972, $56,2232-2252$.

(39) Garret, B. C.; Truhlar, D. G. Accuracy of Tunneling Corrections to Transition State Theory for Thermal Rate Constants of Atom Transfer Reactions. J. Phys. Chem. 1979, 83, 200-203.

(40) Wang, Y.; Li, J.; Yang, Q.; Zhong, C. Two-Dimensional Covalent Triazine Framework Membrane for Helium Separation and Hydrogen Purification. ACS Appl. Mater. Interfaces 2016, 8, 8694-8701.

(41) Pirani, F.; Brizi, S.; Roncaratti, L.; Casavecchia, P.; Cappelletti, D.; Vecchiocattivi, F. Beyond the Lennard-Jones Model: A Simple and Accurate Potential Function Probed by High Resolution Scattering Data Useful for Molecular Dynamics Simulations. Phys. Chem. Chem. Phys 2008, 10, 5489-5503.

(42) Pitonák, M.; Hesselmann, A. Accurate Intermolecular Interaction Energies from a Combination of MP2 and TDDFT Response Theory. J. Chem. Theory Comput. 2010, 6, 168-178.

(43) Robeson, L. M. The Upper Bound Revisited. J. Membrane Sci. 2008, 320, 390-400. 
(44) Lennard-Jones, J. E.; Devonshire, A. F. Diffraction and Selective Adsorption of Atoms at Crystal Surfaces. Nature 1936, 137, 1069-1070.

(45) Sanz, A. S.; Miret-Artés, S. Selective Adsorption Resonances: Quantum and Stochastic Approaches. Physics Reports 2007, 451, 37-154.

(46) Hernández, M. I.; Campos-Martínez, J.; Miret-Artés, S.; Coalson, R. D. Lifetimes of SelectiveAdsorption Resonances in Atom-Surface Elastic Scattering. Phys. Rev. B 1994, 49, 83008309.

(47) Liu, K. Quantum Dynamical Resonances in Chemical Reactions: from A+BC to Polyatomic Systems. Adv. Chem. Phys. 2012, 149, 1-46.

(48) Guo, H.; Farjamnia, A.; Jackson, B. Effects of Lattice Motion on Dissociative Chemisorption: Toward a Rigorous Comparison of Theory with Molecular Beam Experiments. J. Phys. Chem. Lett. 2016, 7, 4576-4584. 
TOC Graphic

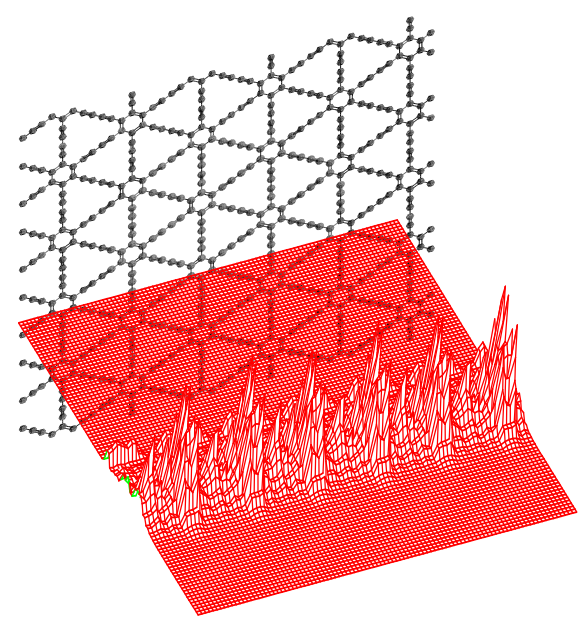

